\title{
OCT assessment of aortic wall degradation for surgical guidance
}

\author{
E. Real*a ${ }^{\mathrm{a}}$, J. F. Val-Bernal ${ }^{\mathrm{c}}$, A. Pontón ${ }^{\mathrm{b}}$, M. Calvo Díez ${ }^{\mathrm{b}}$, M. Mayorga ${ }^{\mathrm{c}}$, J.M. Revuelta ${ }^{\mathrm{b}}$, \\ J. M. López-Higuera a and O. M. Conde ${ }^{\mathrm{a}}$ \\ ${ }^{a}$ University of Cantabria, Dep. TEISA, Photonics Engineering Group, Avda. Los Castros S/N, 39005 \\ Santander, Spain \\ bUniversity of Cantabria and IFIMAV, Cardiovascular Surgery Department, Marqués de Valdecilla \\ University Hospital, Medical Faculty, Avda.Cardenal Herrera Oria S/N 39011, Santander, Spain \\ 'University of Cantabria and IFIMAV, Anatomical Pathology Department, Marqués de Valdecilla \\ University Hospital, Medical Faculty, Avda. Cardenal Herrera Oria S/N 39011, Santander
}

\begin{abstract}
The degradation of the wall in large cardiovascular vessels, such as the aorta artery, induces weakness in the vessel that can lead to the formation of aneurysms and the rupture of the vessel. Characterization of the wall integrity is assessed by OCT for future intraoperative assistance in aneurysm graft surgery interventions. Optical Coherence Tomography (OCT) provides cross sectional images of the wall of the aortic media layer. Wall degradations appear as spatial anomalies in the reflectivity profile through the wall thickness. Wall degradation assessment is proposed by automatic identification and dimensioning of these anomalies within the homogeneous surrounding tissue.
\end{abstract}

Keywords: OCT, thoracic aorta, arterial wall, aneurysm, Gabor wavelet, anomalies automatic identification

\section{INTRODUCTION}

Surgical repair of ascending thoracic aneurysms usually involves the substitution of the diseased portion of the aorta by a fabric tube, or graft, that it is sewn onto both cut ends to replace the portion that is removed. This is performed while a heart-lung machine is connected to the patient to maintain circulation and preventing that no blood flow is present in the aorta [1]. The thoracic aortic graft has to be sewn to aortic tissue adjacent to the aneurysmal one. Graft replacement success correlates with the healthy status of the tissue where the graft is sewn to prevent further complicated medical prognosis related with pseudo-aneurysm formation. Moreover, excising more healthy tissue than needed could become complicated when approaching aortic sections such us the aortic arc.

The aorta is an artery structured in three different layers: intima, media and adventitia starting from the vessel lumen [2]. In large arteries, such as the aorta, the first and third layers are formed mostly from connective tissue (epithelium, and collagen). The media layer provides strength and elasticity, and it is mainly composed by smooth muscle cells (SMC) and elastin. The degradation of SMCs in the media layer produces holes filled by acidic polysaccharides, leading to structural disorders that induce a weakening of the artery and its functions [2]. SMCs degradation ultimately induces the dilation of the artery, what is called an aneurysm. This dilated and weakened artery has higher probability of fissure producing a deadly internal hemorrhage.

Optical Coherence Tomography (OCT) can be applied to visualize tissue within depth of few millimeters, enabling to see a region of the media layer [3]. SMCs and acidic polysaccharides produce different backscattering intensity profiles when imaged with OCT. This can be observed as low reflectivity areas where acidic polysaccharides become located, in contrast with regions of SMCs. Hence, the wall anomaly can be seen as holes in the OCT image.

This work proposes an analysis method focused on detecting these areas of low reflectivity in the OCT image. The amount and extension of anomalies seems to be linearly related to the arterial wall structural wellness. This hypothesis has been contrasted against a semi-quantitative pathological score of the aortic wall condition [4]. Under this situation, aortic samples can be classified into different risk categories according to their artery real degradation. This information is of great help for cardiovascular surgeons when performing graft repair of an aneurysm.

*eusebio.real@unican.es; Phone: 34942 200877+12; gif.teisa.unican.es

23rd International Conference on Optical Fibre Sensors, edited by José Miguel López-Higuera,

Julian Jones, Manuel López-Amo, José Luis Santos, Proc. of SPIE Vol. 9157, 91575H

(C) 2014 SPIE · CCC code: $0277-786 X / 14 / \$ 18 \cdot$ doi: $10.1117 / 12.2059818$ 


\section{MATERIALS \& METHODS}

\subsection{Aortic samples}

The sample set consists of 15 tissue samples coming from human ex-vivo aorta. Samples come from Ascending Thoracic Aorta Aneurysm intervention (12 samples) and from donors for heart transplantations (3 samples). Specimens are classified according to a semi-quantitative grading scheme [4]. This scheme is assessed by expert pathologists that grade stained samples according to different criteria (fibrosis, medionecrosis, cystic medial change, SMC orientation and elastic fiber fragmentation), computing a global histological score for each sample, from 0 (no degradation) to 12 (high degradation).

Samples are later grouped into three different categories according to their histological score. Category 1 , scores 0 to 3 , showing low or no degradation. Category 2, scores 4 to 8 , showing medium degradation. Category 3 , scores 9 to 12, with severe degradation (Fig.1). Category 1 would be ideal for graft placement, while category 3 should be avoided as the structural consistence would be really poor. Furthermore, tissue classified as category 3 should be excised to avoid later medical complications. For this reason, the key point should be the accurate diagnosis of tissue of category 3 .

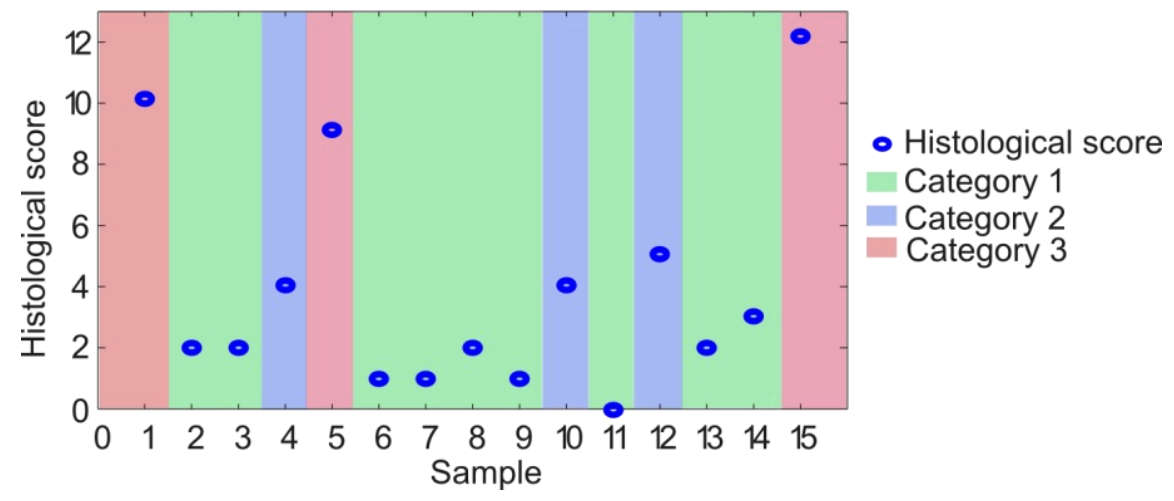

Figure 1. Histological score and group categories for tissue samples.

\subsection{Optical set-up}

The OCT system used is a benchtop Thorlabs OCS1300SS, based on a swept source with a center wavelength of 1325 $\mathrm{nm}$. It provides a resolution in air of $12 \mu \mathrm{m}$ axial and $25 \mu \mathrm{m}$ transverse, giving a maximum penetration in air conditions of 3 and $10 \mathrm{~mm}$ width. The sampling resolution is $512 \times 1024$ pixels and 8 bits for intensity levels digitalization.

Samples are conserved in buffer saline buffer until OCT measurements are performed. Several regions of interest (ROI) are measured on each tissue sample because degradation is not spatially homogeneous along the vessel wall. The total number of ROIs in the dataset is 120. OCT images are taken from the intima layer, focusing the OCT probe on the surface of the sample [3]. After OCT characterization, histological analysis is applied by the pathologists and the samples are graded according the semi-quantitative procedure [4].

\subsection{OCT signal processing}

OCT image processing is aimed at highlighting the areas filled with acidic polysaccharides, seen through OCT as low intensity reflectivity areas. To this aim, the following procedure has been applied:

1. The region of tissue is delimited from the region of air above it, applying the Otsu algorithm for thresholding [5].

2. A wavelet based algorithm [6-7] is used to clean the noise in the image. This algorithm is based on the use of non-orthogonal, complex valued, log-Gabor wavelets that preserve phase information.

3. Aortic wall tissue is automatically segmented to focus the analysis on the media layer. The region of interest is comprehended between $85 \mu \mathrm{m}$ (20 pixels) below surface (intima layer) and 650 $\mu \mathrm{m}$ (150 pixels) in depth (limited by the system response [3]).

4. The segmented tissue is thresholded to identify and isolate low reflectivity areas. The threshold applied is the 5th percentile of the intensity distribution of each image. 
5. Connected regions on the image, below the previous threshold, are taken into account individually. Each tissue section has a number of connected areas, each of them of a specific dimension.

6. The skewness of the distribution of areas size is computed for every image. Each sample is assigned to a category according to this statistical parameter over all their ROIs. As it was mentioned before, degradation is not necessarily seen on every ROI and a joint decision needs to be implemented.

\section{RESULTS AND DISCUSSION}

The proposed procedure evidences the presence of areas of high intensity levels, which has its origin in the reflectivity differences among homogeneous tissue and the degradation of the wall produced in pathological samples. After segmentation of the images and noise reduction, diagnosis can be simplified to a computation of the areas of the anomalies detected in the image (Fig. 2).

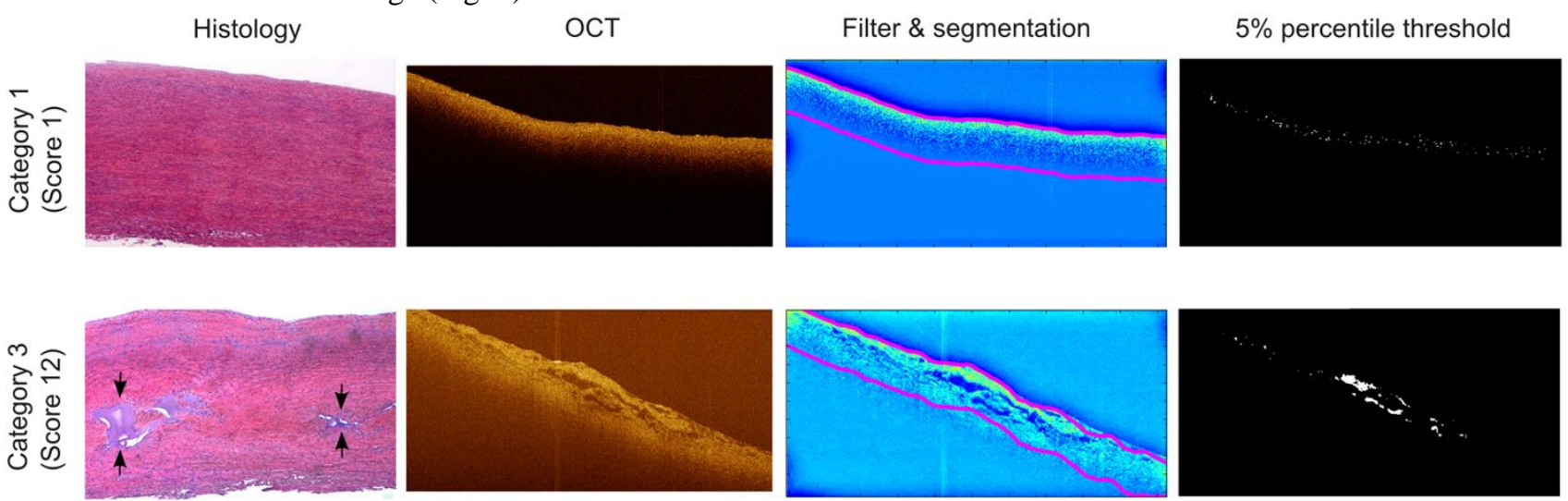

Figure 2. Comparison of two tissue samples of category 1 (upper, low degradation) and category 3 (lower, severe degradation). The histological grading $\left(1^{\text {st }}\right.$ column$)$ is the gold standard for characterization. OCT images (2nd column) are filtered and, after segmentation $\left(3^{\text {rd }}\right.$ column $)$ of the region of analysis, optimal thresholding is applied to highlight degraded areas $\left(4^{\text {th }}\right.$ column).

In low degraded samples, anomalies have small dimension whilst in the cases of high degradation, areas tend to be larger. There are also a significant number of small anomalies, corresponding to regions not so degraded, that leave a medium anomaly area very low, both in degraded and not degraded samples. In the case of severely degraded tissues, large areas deviate the median area of anomalies towards higher values. For this reason, the criterion for classification has been the skewness parameter of the dimension of the detected anomalies (Fig. 3). Furthermore, if the dimension of degraded areas is large, the sample will be highly degraded, but if the ROI is taken on a section of low degradation, the diagnosis could fail. For that reason, the diagnosis takes place using every ROI belonging to each sample.

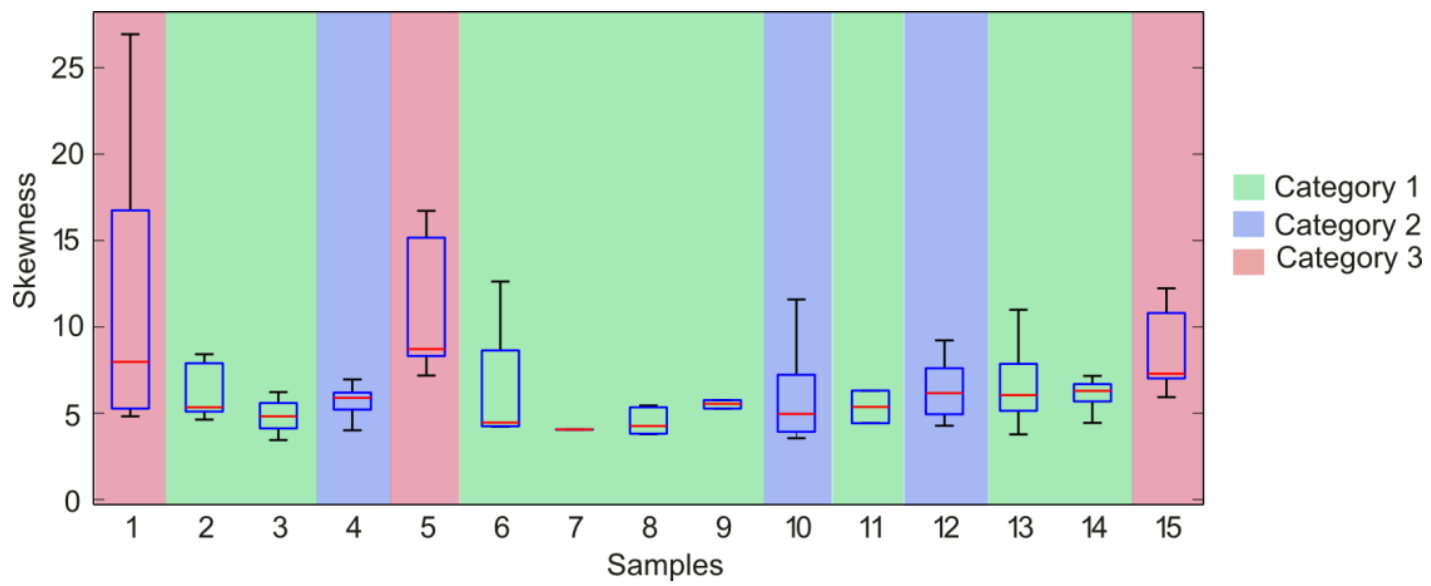

Figure 3. Boxplot of the skewness of the areas detected on each tissue sample. The red line represents the median value. The blue box represents $25^{\text {th }}$ to $75^{\text {th }}$ percentile variation. The black line represents the $99 \%$ percentile. Skewness in more degraded aortic walls (cat\#3, red) becomes larger showing evidences of diagnosis. 
According with results in Fig. 3, different thresholds can be applied to separate samples from the different categories. The most important category to be detected is cat\#3, as this tissue is weak and should be excised. With the proposed method, cat\#3 samples are detected in every occasion (Fig.4). The same happens with cat\#1 which tissue would be optimal for graft placement.

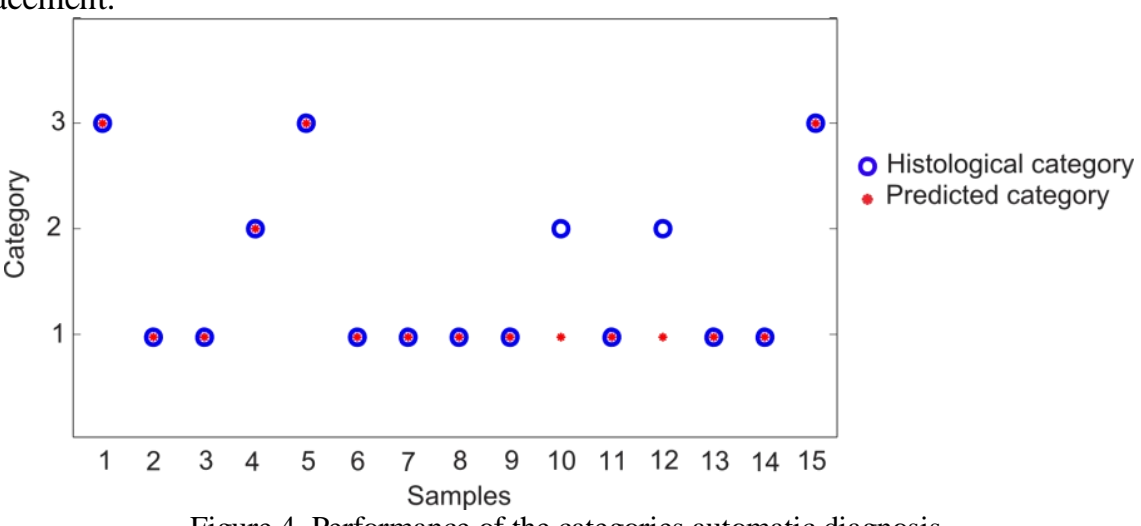

Figure 4. Performance of the categories automatic diagnosis.

\section{CONCLUSION}

An automatic method for degradation diagnosis of the aortic wall has been proposed. The method consists on identifying degraded areas in OCT images obtained from ex-vivo tissue samples of ascending thoracic aortas from aneurysms and donors. The final performance of the method accurately detects the most degraded tissues from a pathological point of view. This technique can help in the case of surgical aneurysm interventions.

\section{ACKNOWLEDGEMENTS}

This work has been supported by the projects DA2TOI (FIS2010-19860), TFS (TEC2010-20224-C02-02).

\section{REFERENCES}

[1] Hiratzka, L.F, et al., "Guidelines for the diagnosis and management of patients with thoracic aortic disease: executive summary," J Am Coll Cardiol 55, 1509-1544 (2010).

[2] Rizzo, R.J., et al., "Collagen types and matrix protein content in human abdominal aortic aneurysms,"J Vasc Sueg 10(4), 365-373 (1989).

[3] Real, E., Eguizábal, A., Pontón, A., Calvo-Díez,M., Val-Bernal, J. F., Mayorga, M., Revuelta, J.M., LópezHiguera, J.M. and Conde, O.M.," Optical coherence tomography assessment of vessel wall degradation in thoracic aortic aneurysms," J Biomed Opt 18(12),106003 (2013).

[4] Bechtel J.F.M., Noack, F., Sayk, F., Erasmi, A.W., Bartels and C., Sievers, H.H., "Histopathological grading ofascending aortic aneurysm: comparison of patients with bicuspid versus tricuspid aortic valve," J Heart Valve Dis 12(1), 54-61 (2003).

[5] Otsu, N.,"A Threshold Selection Method from Gray-Level Histograms," IEEE Sys Man Cyb 9(1), $62-66$ (1979).

[6] Kovesi,P., "Phase Preserving Denoising of Images," The Australian Pattern Recognition Society Conference: DICTA'99 212-217 (1999)

[7] Kovesi,P.," MATLAB and Octave Functions for Computer Vision and Image Processing," Csse,May 2000, $<$ http://www.csse.uwa.edu.au/ pk/research/matlabfns/>, (12 November 2013) 